\title{
Nonspecific interstitial pneumonia: A rare adverse reaction of atorvastatin
}

\author{
Jack Xu*1,2, Steven Verga ${ }^{1,2}$, Jonathan Stoll ${ }^{1,2}$, Lauren Pioppo ${ }^{1,2}$, Eileen Shanahan², Wajahat Khan ${ }^{2}$ \\ ${ }^{1}$ Department of Internal Medicine, Rutgers Robert Wood Johnson Medical School, New Brunswick, NJ, United States \\ ${ }^{2}$ Penn Medicine Princeton Health, Plainsboro, NJ, United States
}

Received: July 29, 2018

DOI: $10.5430 /$ crim.v5n4p16
Accepted: September 13, $2018 \quad$ Online Published: September 27, 2018

URL: https://doi.org/10.5430/crim.v5n4p16

\begin{abstract}
Introduction: Statins have been shown to effectively prevent both cardiovascular morbidity and mortality by inhibiting the hydroxymethylglutaryl coenzyme A (HMG-CoA) reductase enzyme. Pulmonary complications are very rare, and can include pneumonitis, pleural effusion, and nonspecific interstitial pneumonia (NSIP). There have been very few previously documented cases of statin-induced fibrotic NSIP.

Case report: We present a female with a history of hyperlipidemia on atorvastatin who presented with shortness of breath. Computed tomography scan of the chest revealed interstitial infiltrates with bilateral ground-glass opacities. She underwent a surgical lung biopsy which showed uniform fibrous alveolar septal thickening, scattered collections of alveolar macrophages and inflammation, along with areas of fibrosis. The findings were most suggestive of fibrotic NSIP. Atorvastatin was stopped and she was started on mycophenolic acid with improvement of her symptoms.

Discussion: Although rare, the clinician should be aware of possible pulmonary complications of statin therapy. The exact mechanism of injury is unclear, however immunological or toxicological mechanisms are implicated. One case series of statin induced interstitial lung disease showed some improvement of dyspnea with systemic glucocorticoids and termination of statin therapy. Statins, specifically pravastatin, lovastatin, and simvastatin have been associated with drug induced pneumonitis and interstitial lung disease.
\end{abstract}

Key Words: Nonspecific interstitial pneumonia

\section{INTRODUCTION}

Statins have been shown to effectively prevent both cardiovascular morbidity and mortality by inhibiting the hydroxymethylglutaryl coenzyme A (HMG-CoA) reductase enzyme. ${ }^{[1]}$ They increase the number of low-density lipoprotein receptors in the liver and as a result, decrease serum low-density lipoprotein cholesterol. They are usually tolerated well clinically and their major adverse effects include hepatotoxicity and myotoxicity. ${ }^{[2]}$ Since their introduction in the 1980s, they have become the most prescribed cholesterollowering drugs. With their widespread use and availability, more reports of other adverse effects including pulmonary toxicity are being reported. ${ }^{[2]}$ Pulmonary complications are very rare, and can include hypersensitivity pneumonitis, pleural effusion, and nonspecific interstitial pneumonia (NSIP). ${ }^{[2-10]}$ There has only been one previously documented case of statin-induced fibrotic NSIP. ${ }^{[2]}$

${ }^{*}$ Correspondence: Jack Xu, M.D.; Email: jaxu54@gmail.com; Address: Department of Internal Medicine, Rutgers Robert Wood Johnson Medical School, New Jersey, United States. 


\section{CASE REPORT}

We present a 73-year-old female with a history of hypertension, diabetes, and hyperlipidemia who presented with a several month history of shortness of breath. She reported progressively worsening exercise tolerance as well as a chronic cough without hemoptysis. There was no history of connective tissue disease. Home medications included atorvastatin, lisinopril, metformin, fenofibrate, aspirin, and verapamil. On room air, her oxygen saturation was $92 \%$. Chest examination was remarkable for diminished breath sounds bilaterally. Computed tomography scan of the chest revealed interstitial infiltrates with bilateral ground-glass opacities (see Figure 1). Due to her progressive diffuse interstitial lung disease, she underwent a surgical lung biopsy which showed uniform fibrous alveolar septal thickening, scattered collections of alveolar macrophages and inflammation, along with mild areas of fibrosis (see Figure 2). The findings were most suggestive of fibrotic NSIP. Since the patient did not have a history of connective tissue disease or other infections, druginduced pneumonitis was highly suspected. Even though the patient had been on atorvastatin for years, it was stopped and the patient was started on mycophenolic acid with improvement in her symptoms over six months. She was started on mycophenolic acid rather than steroids because she was deemed a poor candidate for steroids due to her diabetes and obesity.

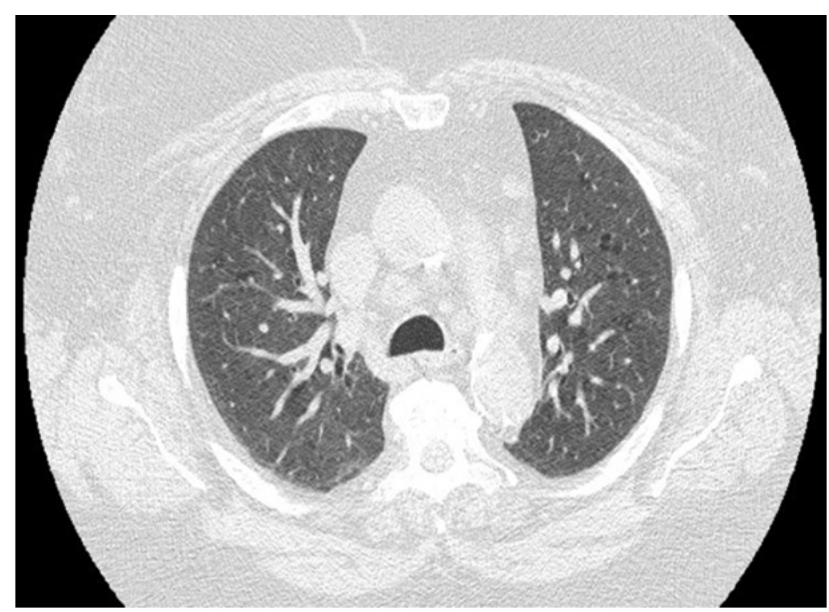

Figure 1. High resolution CT scan of the chest showing upper lung cystic changes

\section{Discussion}

Statins are increasingly prescribed to reduce cholesterol and improve cardiovascular health. ${ }^{[1]}$ They are among the most widely prescribed drugs in the world due to their low frequency of side-effects. Although rare, the clinician should be aware of possible pulmonary complications of statin therapy.
The etiology of NSIP can be idiopathic or can be seen in association with connective tissue disease, human immunodeficiency, virus, medication-induced, or hypersensitivity pneumonitis. Investigating the etiology of NSIP is important as it guides management of the disease, since in medicationinduced NSIP, removal of the inciting exposure alone may be adequate treatment. ${ }^{[3]}$

The exact mechanism of drug-induced NSIP is unclear, however immunological or toxicological mechanisms are implicated. ${ }^{[2]}$ Statins may have a toxic effect leading to activation of an inflammatory immune response, possibly mediated by the inhibition of phospholipadases and thus leading to cellular accumulation of intralysosomial phospholipids. It is uncertain as to why this effect is only seen in so few of the millions of patients who are on statins but it may be possible that this unique reaction may be due to genetic or other predisposing factors. The timing of the onset of pulmonary symptoms is irregular as some patients had been taking statin therapy for months to years before developing symptoms. ${ }^{[7]}$

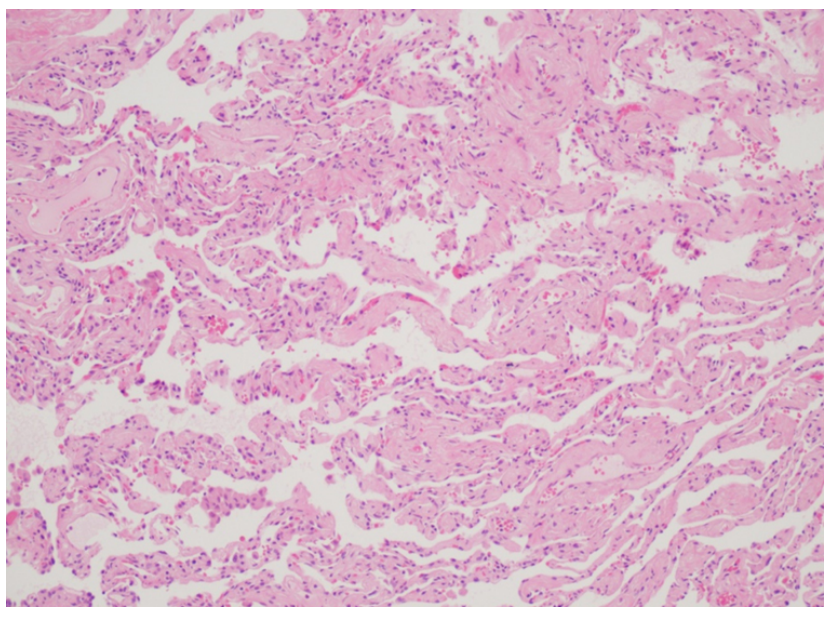

Figure 2. Biopsy of the right middle and upper lobes showing uniform fibrous alveolar septal thickening, suggestive of non-specific interstitial pneumonia, fibrosing variant

One case series of statin induced interstitial lung disease showed some improvement of dyspnea with systemic glucocorticoids and termination of statin therapy. ${ }^{[3]}$ Statins, specifically pravastatin, lovastatin, and simvastatin have been associated with drug induced pneumonitis and interstitial lung disease. ${ }^{[2]}$

Being able to identify rare side effects of commonly used medications is a difficult task for the clinician especially when symptoms may not present until months to years after starting the medication. However, failure to recognize potential causes of NSIP may be fatal, so we believe that clinicians 
should consider cessation of statins in patients who present with NSIP. We hope to bring awareness of the possible link between statins in NSIP and that more studies regarding this potential association will be done.

\section{CONFlicts OF INTEREST Disclosure}

The authors declare no conflicts of interest.

\section{REFERENCES}

[1] Ballantyne CM, Grundy SM, Oberman A, et al. Hyperlipidemia: diagnostic and therapeutic perspectives. J Clin Endocrinol Metab. 2000 85: 2089. PMid:10852435. https://doi.org/10.1210/jcem.8 5.6.6642-1

[2] Lantuejoul S, Brambilla E, Brambilla C, et al. Statin-induced fibrotic nonspecific interstitial pneumonia. Eur Respir J. 2002; 19: 577. PMid:11936540. https://doi.org/10.1183/09031936.02.00 258802

[3] Liebhaber MI, Wright RS, Gelberg HJ, et al. Polymyalgia, hypersensitivity pneumonitis and other reactions in patients receiving HMG-CoA reductase inhibitors: A report of ten cases. Chest. 1999; 115: 886-889. PMid:10084510. https://doi.org/10.1378/ch est.115.3.886

[4] Huang LK, Tsai MJ, Tsai HC, et al. Statin-induced lung injury: diagnostic clue and outcome. Postgrad Med J. 2013; 89 (1047): 14-9. PMid:23043128. https://doi.org/10.1136/postgradmedj-2 011-130209

[5] De Groot REB, Willems INA, Dijkman HJ. Interstitial lung disease with pleural effusion caused by simvastatin. J Intern Med. 1996;
239: 361-363. PMid:8774391. https://doi.org/10.1046/j.13 65-2796.1996.411762000.x

[6] Saad N, Camus P, Suissa S, et al. Statins and the risk of interstitial lung disease: a cohort study. Thorax. 2013; 68: 363-364. PMid:23299962. https://doi.org/10.1136/thoraxjnl-201 2-201823

[7] Walker T, McCaffery J. Steinfort C. Potential link between HMGCoA reductase inhibitor (statin) use and interstitial lung disease. Med J Aust. 2007; 186: 91-4. PMid:17223772.

[8] Veyac G, Cellerin L, Jolliet P. A case of interstitial lung disease with atorvastatin (Tahor) and a review of the literature about these effects observed under statins. Therapie. 2006; 61: 57-67.

[9] Liscoet-Loheac N, Andre N, Couturaud F, et al. Hypersensitivity pneumonitis in a patient taking pravastatin. Rev Mal Respir. 2001; 18 (4 Pt 1): 426-8. PMid:11547251.

[10] Fernandez AB, Karas RH, Alsheikh-Ali AA, et al. Statins and interstitial lung disease: a systematic review of the literature and of food and drug administration adverse event reports. Chest. 2008; 134: 824-30. PMid:18689579. https://doi.org/10.1378/chest.08-0943 